\title{
MEANS OF ITERATED TRANSFORMATIONS IN REFLEXIVE VECTOR SPACES*
}

\author{
EDGAR R. LORCH
}

If $U$ is a unitary transformation in a Hilbert space, the convergence of the sequence of transformations $\left\{(1 / n) \sum_{s=0}^{n-1} U^{s}\right\}$ to a projection $P$ has been established by von Neumann in his proof of the quasiergodic hypothesis. $\dagger$ Recently, the question of the existence of mean ergodic theorems in more general spaces than Hilbert space has received attention. One is here concerned with a bounded linear transformation $V$ of bound not greater than 1 , that is, $|V| \leqq 1$, defined over a Banach space $\mathfrak{B}$, or more generally with a transformation $V$ whose iterates $V^{n}, n=0,1,2, \cdots,\left(V^{0}=I\right)$ are uniformly bounded: $\left|V^{n}\right| \leqq K$. The problem then is to characterize the spaces $\mathfrak{B}$ for which the arithmetic means $T_{n}=(1 / n) \sum_{s=0}^{n-1} V^{s}$ converge, and to examine the nature of the limiting transformation. The case $\mathfrak{B}=L^{p}, p>1$, has been treated by F. Riesz.\$ Very recently, Garrett Birkhoff, considering transformations of bound not greater than 1, has extended Riesz' results to uniformly convex spaces. § Our Theorem 2 includes all these results as special cases. $\|$ The methods we use resemble closely at certain points those used in a Hilbert space. The properties of reflexive spaces $\uparrow$ to which appeal is made are simple and are examples of the elegant reciprocity relations which characterize those spaces (see, for instance, Theorem 1).

The adjoint space of a space $\mathfrak{B}$ is denoted by (B). The adjoint of

* Presented to the Society, September 8, 1939. Abstracted in this Bulletin, abstract 45-7-270 (1939).

$\dagger \mathrm{J}$. von Neumann, Proof of the quasi-ergodic hypothesis, Proceedings of the National Academy of Sciences, vol. 18 (1932), pp. 70-82.

$\ddagger$ F. Riesz, Some mean ergodic theorems, Journal of the London Mathematical Society, vol. 13 (1938), pp. 274-278.

$\S$ Garrett Birkhoff, this Bulletin, abstract 45-1-77 (1939); Duke Mathematical Journal, vol. 5 (1939), pp. 19-20; J. A. Clarkson, Uniformly convex spaces, Transactions of this Society, vol. 40 (1936), pp. 396-414.

\| The results of this note were communicated orally by the author to Professor B. O. Koopman in the spring of 1938 . It had been planned originally to delay announcement and publication until certain other researches had been completed. But since theorems of the type treated here have received considerable attention recently, it was decided to publish this proof separately without further delay.

I See the author's On a calculus of operators in reflexive spaces, Transactions of this Society, vol. 45 (1939), pp. 217-234. A discussion of the elementary properties of manifolds in reflexive spaces and of projections in any Banach space is found in Chapter II. 
$(\mathfrak{B})$ is then $((\mathfrak{B}))$. The relation $\mathfrak{B} \subset((\mathfrak{B}))$ expresses the fact that a certain obvious isometric mapping carries $\mathfrak{B}$ into a subset of $((\mathfrak{B}))$. If $\mathfrak{B}=((\mathfrak{B}))$, the space $\mathfrak{B}$ is called reflexive. More precisely, if beginning with a space $\mathfrak{B}$, a $\Phi \varepsilon((\mathfrak{B}))$ be chosen, then $\mathfrak{B}$ is reflexive if there exists an $f \varepsilon \mathfrak{B}$ such that $\Phi(F)=F(f)$ for all $F \varepsilon(\mathfrak{B})$. The spaces $L^{p}$, $p>1$, are well known to be reflexive. The fact that any uniformly convex space is reflexive has recently been demonstrated.* If $\mathfrak{M}$ is a closed linear manifold (c.l.m.) in $\mathfrak{B}, \mathfrak{M}^{\perp}$ denotes the c.l.m. in ( $\mathfrak{B}$ ) consisting of all $F \varepsilon(\mathfrak{B})$ orthogonal to every $f \varepsilon \mathfrak{M}$. If $\mathfrak{B}$ is reflexive, $\mathfrak{M}^{\perp \perp}=\mathfrak{M} . \dagger$

A bounded linear transformation (b.l.t.) $P$ in any $\mathfrak{B}$ is called a projection if $P^{2}=P$. If $P$ is a projection, there exist two c.l.m. $\mathfrak{M}$ and $\mathfrak{N}$ with $P g=g$ if $g \varepsilon \mathfrak{M}, P h=0$ if $h \varepsilon \mathfrak{N}$ and for every $f \varepsilon \mathfrak{B}$, unique $g \varepsilon \mathfrak{M}$, $h \varepsilon \mathfrak{R}$ exist such that $f=g+h$. Conversely, manifold pairs of the type just described generate a projection. $\dagger$

Let $\overline{\mathrm{T}}$ be the adjoint of a b.l.t. $T$. Then it is known that $|\bar{T}|=|T|$. The principal result of this note will be proved with the assistance of the following theorem.

THEOREM 1. Let $\mathfrak{B}$ be a reflexive vector space. Let $T$ be a bounded linear transformation in $\mathfrak{B}$. Let $\mathfrak{M}$ denote the closed linear manifold of elements $f$ for which $T f=0$; let $\mathfrak{N}$ denote the closed linear manifold spanned by the elements $T f, f \varepsilon \mathfrak{B}$. Let $\bar{T}$ be the adjoint of $T$, and let (M) and $(\mathfrak{N})$ be the manifolds associated with $\bar{T}$ in the manner just indicated. Then $(\mathfrak{M})=\mathfrak{N}^{\perp},(\mathfrak{N})=\mathfrak{M}^{\perp}, \mathfrak{M}=(\mathfrak{N})^{\perp}, \mathfrak{N}=(\mathfrak{M})^{\perp}$.

By definition, $\bar{T} F=G$ with $F, G \varepsilon(\mathfrak{B})$, if $F(T f)=G f$ for all $f \varepsilon \mathfrak{B} . \ddagger$ Let $F \varepsilon(\mathfrak{M})$; then $\bar{T} F=0$ or $F T f=0$, hence $F \perp \mathfrak{N}, F \varepsilon \mathfrak{N}^{\perp}$; thus $(\mathfrak{M}) \subset \mathfrak{N}^{\perp}$. Conversely, let $F \varepsilon \mathfrak{N}^{\perp}$; then $F(T f)=0, \bar{T} F=0, F \varepsilon(\mathfrak{M})$ and $\mathfrak{N}^{\perp} \subset(\mathfrak{M})$. Thus $(\mathfrak{M})=\mathfrak{N}^{\perp}$. Now let $F \varepsilon(\mathfrak{N})$; then for $\epsilon>0$ there exists an $F^{\prime}$ with $\left\|F-\bar{T} F^{\prime}\right\| \leqq \epsilon$. If $f \varepsilon \mathfrak{M}, \bar{T} F^{\prime} f=F^{\prime} T f=0$; thus $\bar{T} F^{\prime} \perp \mathfrak{M}, \bar{T} F^{\prime} \varepsilon \mathfrak{M}^{\perp}$. Since $\epsilon$ is arbitrary, $F \varepsilon \mathfrak{M}^{\perp}$ or $(\mathfrak{l}) \subset \mathfrak{M}^{\perp}$. Suppose $(\mathfrak{N}) \neq \mathfrak{M}^{\perp}$, then there exists an $F \varepsilon \mathfrak{M}^{\perp}$ such that $F \notin(\mathfrak{N})$. Since $\mathfrak{B}$ is reflexive, there exists an $f \varepsilon \mathfrak{B}$ such that $F f=1, f \perp(\mathfrak{N})$, or $\bar{T} G f=G T f$

* B. J. Pettis, this Bulletin, abstract 44-7-295 (1938); Duke Mathematical Journal, vol. 5 (1939), pp. 249-253; D. Milman, On some criteria for the regularity of spaces of the type (B), Comptes Rendus de l'Académie des Sciences de l' URSS, vol. 20 (1938), pp. 243-246. This author uses the terminology originally introduced by H. Hahn (Über lineare Gleichungssysteme in linearen Raïmen, Journal für die reine und angewandte Mathematik, vol. 157 (1927), pp. 214-229) and calls a reflexive space "regular."

$\dagger$ Lorch, loc. cit.

‡ S. Banach, Théorie des Opérations Linéaires, p. 100. 
$=0$ for all $G \varepsilon(\mathfrak{B})$. Hence $T f=0, f \varepsilon \mathfrak{M}, F f=0$ and not 1 . This means that $(\mathfrak{N})=\mathfrak{M}^{\perp}$.

Since $\mathfrak{N}^{\perp}=(\mathfrak{M}), \mathfrak{R}^{\perp \perp}=\mathfrak{N}=(\mathfrak{M})^{\perp}$. Similarly, since $\mathfrak{M}^{\perp}=(\mathfrak{R}), \mathfrak{M}^{\perp \perp}$ $=\mathfrak{M}=(\mathfrak{N})^{\perp}$.

THEOREM 2. Let $\mathfrak{B}$ be a reflexive vector space. Let $V$ be a bounded linear transformation in $\mathfrak{B}$ such that $\left|V^{n}\right| \leqq K, n=0,1,2, \cdots$. Then the sequence $\left\{T_{n}\right\}$ where $T_{n}=(1 / n) \sum_{s=0}^{n-1} V^{s}$ converges strongly to a limiting transformation $P$ which is a projection. The relation $P f=f$ holds precisely for those elements $f$ for which $V f=f$. The relation $P f=0$ holds precisely for the elements of the closed linear manifold spanned by elements of the form $V g-g, g$ arbitrary in $\mathfrak{B}$. The bound of $P$ satisfies the inequality $|P| \leqq K$.

Let $\mathfrak{M}$ be the c.l.m. of elements $f$ for which $V f=f$. Then $T_{n} f=f \rightarrow f$.

Let $\mathfrak{N}$ denote the c.l.m. spanned by elements of the form $V g-g$, $g$ arbitrary in $\mathfrak{B}$. If $f \boldsymbol{\varepsilon} \mathfrak{N}$ and $\epsilon>0$ is arbitrary, then $g, h \varepsilon \mathfrak{B}$ may be found such that $f=V g-g+h$ with $\|h\| \leqq \epsilon$. Now $T_{n} f=(1 / n)\left(V^{n} g-g\right)$ $+T_{n} h$. Furthermore $\left|T_{n}\right| \leqq(1 / n) \sum_{s=0}^{n-1}\left|V^{s}\right| \leqq K$; therefore $\left\|T_{n} f\right\|$ $\leqq(2 / n) K\|g\|+K \epsilon \leqq 2 K \epsilon$ providing $n$ is sufficiently large. Thus $T_{n} f \rightarrow 0$ when $f \varepsilon \mathfrak{\imath}$.

Now let $f \varepsilon \mathfrak{M}, g \varepsilon \mathfrak{R}$; then $\|f\| \leqq K\|f+g\|$. This follows from $\left|T_{n}\right| \leqq K$ and $T_{n}(f+g) \rightarrow f$. As a consequence the only element common to $\mathfrak{M}$ and $\mathfrak{N}$ is 0 . In addition, the totality of elements of the form $f+g$ is a c.l.m. which will be designated by $\mathfrak{M}+\mathfrak{+} \mathfrak{N}$. The strong limit of $T_{n}$ exists in this c.l.m. and is a b.l.t. $P$ of bound $|P| \leqq K$. Clearly $P^{2}=P$ in $\mathfrak{M}+\mathfrak{*}$. To complete the proof, it must be shown that $\mathfrak{M}+\mathscr{+} \mathfrak{N}=\mathfrak{B}$. This equation will be established with the aid of the assumption, not employed up to this point, that $\mathfrak{B}$ is reflexive.

If $V$ is the adjoint of $\bar{V}$, then $\bar{V}^{n}=\overline{\left(V^{n}\right)}$ and $\left|\bar{V}^{n}\right| \leqq K$. Thus what has just been established for $V$ may be elaborated for $\bar{V}$. Let (M) and $(\mathfrak{N})$ denote the manifolds found for $\bar{V}$ by the method just applied to $V$. Applying Theorem 1 to the transformations $V-I$ and $\bar{V}-\bar{I}$ $=\overline{(V-I)}$, we have $(\mathfrak{M})=\mathfrak{N}^{\perp},(\mathfrak{N})=\mathfrak{M}^{\perp}$. If $f \varepsilon \mathfrak{B}$ but $f \notin \mathfrak{M}+\mathfrak{*} \mathfrak{N}$, there exists an $F \varepsilon(\mathfrak{B})$ such that $F f=1, F g=0$ for $g \varepsilon \mathfrak{M}+\mathfrak{*} \mathfrak{R}$. Hence $F \perp \mathfrak{M}$, $F \perp \mathfrak{N}$, or $F \varepsilon \mathfrak{M}^{\perp}=(\mathfrak{N}), F \varepsilon \mathfrak{N}^{\perp}=(\mathfrak{M})$. As $(\mathfrak{M})$ and $(\mathfrak{N})$ have only the element 0 in common, $F=0$, which contradicts $F f=1$. Thus $\mathfrak{M}+\mathfrak{A}$ $=\mathfrak{B}$.

Barnard College, Columbia University 